Pacific Journal of Mathematics

LINEAR ISOMETRIES OF SOME FUNCTION SPACES 


\title{
LINEAR ISOMETRIES OF SOME FUNCTION SPACES
}

\author{
N. V. RAO AND A. K. ROY
}

The purpose of this paper is to describe the linear isometries of the Banach algebras of continuously differentiable functions, Lipschitz functions and absolutely continuous functions on the unit interval with the norms described below. It is also shown how these results continue to hold for more general norms which come from compact convex bodies in $\mathrm{C}^{2}$ satisfying certain properties.

1. Generalities. Throughout this paper we denote by $I$ the unit interval $[0,1]$ and by $\lambda$, Lebesgue measure on $I$. Following customary usage, we denote by

(i) $\mathscr{C}^{1}(I)$ the space of continuously differentiable functions on $I$, with norm $\|f\|=\|f\|_{\infty}+\left\|f^{\prime}\right\|_{\infty}\left(f \in \mathscr{C}^{1}(I)\right)$,

(ii) $\mathscr{L}_{\% / 2}(I)$ the space of Lipschitz functions (Lipschitz of order one with respect to the standard Euclidean metric on $I$ ), with norm $\|f\|=\|f\|_{\infty}+\left\|f^{\prime}\right\|_{\infty}\left(f \in \mathscr{L}_{i / 2}(I)\right)$,

and (iii) $\mathscr{A} \mathscr{C}(I)$ the space of absolutely continuous functions, with norm $\|f\|=\|f\|_{\infty}+\left\|f^{\prime}\right\|_{1}(f \in \mathscr{A} \mathscr{C}(I)) \cdot\left(L^{1}(I)\right.$ and $L^{\infty}(I)$ are of course defined with respect to $\lambda)$.

These function spaces are, as is well-known, Banach algebras under the norms just defined and we have the following inclusions amongst these algebras:

$$
\mathscr{C}^{1}(I) \subset \mathscr{L} / \frac{1}{4}(I) \subset \mathscr{A} \mathscr{C}(I) \text {. }
$$

Our objective in this paper is to determine the linear isometries ${ }^{\mathrm{I}}$ of these spaces. More specifically, we will show in $\S 2$ that all the isometries of $\mathscr{A C}(I)$ are induced by monotone absolutely continuous mappings of the unit interval onto itself whereas the isometries of $\mathscr{L} / 2,(I)$ and $\mathscr{C}^{1}(I)$ come only from the functions $x$ and $1-x$ (§3 and §4, respectively). In the last section, we indicate that precisely the same results continue to hold for isometries under more general norms. We have preferred to discuss the Banach algebra norms first because the main ideas of this generalization are already present there.

The results for the algebra $\mathscr{A} \mathscr{C}(I)$ were first proved in Cambern's paper [1]. We present here a different and perhaps more elementary proof. Cambern also discusses the isometries of $\mathscr{C}^{1}(I)$ with a norm somewhat simpler than the one we use in $\S 4$.

${ }^{1}$ By a linear isometry (or isometry for short) of a Banach space $X$, we mean a linear, norm-preserving transformation of $X$ onto itself. 
We would like to thank Dr. M. H. Vasavada for some interesting conversations about the problems discussed in this work.

2. The algebra $\mathscr{A} \mathscr{C}(I)$. For this section, the norm of $f \in$ $\mathscr{A C}(I)$ is

$$
\|f\|=\|f\|_{\infty}+\left\|f^{\prime}\right\|_{1} \text {. }
$$

Following [1], we embed $\mathscr{A} \mathscr{C}(I)$ as a subspace of $\mathscr{C}(X), X$ a compact Hausdorff space, in the following way. If $V$ denotes the unit ball of $L^{\infty}(I)$, then $V$ is compact in the weak topology induced by $L^{1}(I)$ and we let $X$ be the space $I \times V$ with the product topology. If $f \in \mathscr{A} \mathscr{C}(I)$ and $(x, \alpha) \in X$, defining

$$
\widetilde{f}(x, \alpha)=f(x)+\int_{I} f^{\prime} \bar{\alpha} d \lambda,
$$

it is obvious that $f \rightarrow \widetilde{f}$ gives an isometric isomorphism between $\mathscr{A} \mathscr{C}(I)$ and a closed subspace $Y \subset \mathscr{C}(X)$. We now describe all the extreme functionals in the unit ball of $Y^{*}$. (Only a partial description of these functionals is given in [1]). By [2; page 441], all the extreme functionals are contained in

$$
\left\{e^{i \eta} L(x, \alpha): \eta \in[-\pi, \pi],(x, \alpha) \in I \times V\right\} .
$$

For the statement of the next lemma, we adopt the following notation. If $\varepsilon>0$,

$$
C_{1 \varepsilon}=\left\{z \in \mathrm{C}:|z|=1,|\arg z| \leqq \frac{\pi}{2}+\varepsilon\right\}
$$

and

$$
C_{2 \varepsilon}=\left\{z \in \mathrm{C}:|z|=1,|\arg z| \geqq \frac{\pi}{2}-\varepsilon\right\}
$$

Lemma 2.1. If $\left(x_{0}, \alpha_{0}\right) \in I \times V$ and $0<x_{0}<1$, then the functional $L\left(x_{0}, \alpha_{0}\right) \in \mathscr{A C C}^{*}(I)\left(\equiv Y^{*}\right)$ defined by

$$
L_{\left(x_{0}, \alpha_{0}\right)}(f)=f\left(x_{0}\right)+\int_{I} f^{\prime} \bar{\alpha}_{0} d \lambda,(f \in \mathscr{A} \mathscr{C}(I))
$$

is extreme in the unit ball of $\mathscr{A C}^{*}(I)$ if and only if

(i) $\left|\alpha_{0}\right|=1$ a.e. on $I$ and

(ii) $\quad \forall^{\prime} \varepsilon>0, \lambda\left[\alpha_{0}^{-1}\left(C_{1 \varepsilon}\right) \cap\left(x_{0}-\varepsilon, x_{0}\right)\right]>0$ and

$$
\lambda\left[\alpha_{0}^{-1}\left(C_{2 \varepsilon}\right) \cap\left(x_{0}, x_{0}+\varepsilon\right)\right]>0 .
$$

If $x_{0}$ is 1 (resp. 0) then $\forall \varepsilon>0, \lambda\left[\alpha_{0}^{-1}\left(C_{1 \varepsilon}\right) \cap(1-\varepsilon, 1)\right]>0$ (resp. $\left.\alpha_{0}^{-1}\left[\left(C_{2 \varepsilon}\right) \cap(0, \varepsilon)\right]>0\right)$. 
Proof. Clearly, condition (i) is necessary because $\alpha_{0} \in V$ is extreme if and only if $\left|\alpha_{0}\right|=1$ a.e. so assuming $\left\{\alpha_{0}\right\}=1$ a.e. and $0<x_{0}<1$, let

$$
L_{\left(x_{0}, \alpha_{0}\right)}=\frac{1}{2}\left(L_{1}+L_{2}\right) \text {, }
$$

where $L_{i}(i=1,2)$ belongs to the unit ball of $\mathscr{A}^{C} \mathscr{C}^{*}(I)$. Since $L_{\left\langle x_{0}, \alpha_{0}\right)}(1)=1$, it follows that $L_{1}(1)=L_{2}(1)=1$. By means of the isometric map $f \rightarrow\left(f, f^{\prime}\right)$, we can regard $\mathscr{A} \mathscr{C}(I)$ as a closed subspace of the direct sum $\mathscr{C}(I) \oplus L^{1}(I)$ with the norm

$$
\|(f, g)\|=\|f\|_{\infty}+\|g\|_{1} \text {. }
$$

Extending $L_{i}$ to the whole of $\mathscr{C}(I) \oplus L^{1}(I)$ by means of the HahnBanach theorem, we see easily that $L_{i}$ has the following form:

$$
L_{i}(f)=\int_{I} f d \mu_{i}+\int_{I} f^{\prime}(y) \overline{g_{i}(y)} d \lambda(y),
$$

where $\mu_{i}$ is a nonnegative measure on $I$ with total mass 1 and $g_{i} \in$ $L^{\infty}(I)$ with $\left\|g_{i}\right\|_{\infty} \leqq 1(i=1,2)$. Therefore for all $f \in \mathscr{A} \mathscr{C}(I)$,

$$
f\left(x_{0}\right)+\int_{I} f^{\prime}(y) \overline{\alpha_{0}(y)} d \lambda(y)=\int_{I} f d \mu+\int_{I} f^{\prime}(y) \overline{g(y)} d \lambda(y),
$$

where $\mu=\frac{1}{2}\left(\mu_{1}+\mu_{2}\right)$ is a nonnegative measure on $I$ with total mass 1 and $g=\frac{1}{2}\left(g_{1}+g_{2}\right) \in L^{\infty}(I),\|g\|_{\infty} \leqq 1$, and hence

$$
\begin{aligned}
\int_{I} f^{\prime}(y) \overline{\alpha_{0}}(y) d \lambda(y) & =\int_{I}\left(f(t)-f\left(x_{0}\right)\right) d \mu(t)+\int_{I} f^{\prime}(y) \overline{g(y)} d \lambda(y) \\
& \left.=\int_{I}\left\{\int_{\left[x_{0}, t\right]} f^{\prime}(y) d \lambda(y)\right)\right\} d \mu(t)+\int_{I} f^{\prime}(y) \overline{g(y)} d \lambda(y) \\
& =\int_{I}\left\{\int_{I} C_{\left[x_{0}, t\right]}(y) d \mu(t)\right\} f^{\prime}(y) d \lambda(y)+\int_{I} f^{\prime}(y) \overline{g(y)} d \lambda(y),
\end{aligned}
$$

where

$$
\begin{aligned}
C_{\left[x_{0}, t\right]} & =\text { characteristic function of }\left[x_{0}, t\right] \text { if } t \geqq x_{0} \\
& =- \text { characteristic function of }\left[t, x_{0}\right] \text { if } t<x_{0} .
\end{aligned}
$$

If we define

$$
\beta(y)=\int_{I} C_{\left[x_{0}, t\right]}(y) d \mu(t),
$$

it immediately follows from the above that

$$
\bar{\alpha}_{0}(y)=\beta(y)+\bar{g}(y) \text { a.e. }
$$

Since

$$
\begin{aligned}
\beta(y) & =-\mu[0, y] \text { if } y<x_{0} \\
& =\mu[y, 1] \text { if } y>x_{0},
\end{aligned}
$$


we see that

$$
\begin{aligned}
\bar{g}(y) & =\bar{\alpha}_{0}(y)+\mu[0, y] \text { a.e. if } y<x_{0} \\
& =\bar{\alpha}_{0}(y)-\mu[y, 1] \text { a.e. if } y>x_{0} .
\end{aligned}
$$

Suppose now that condition (ii) of the lemma holds. Let $\varepsilon_{n}$ be any sequence such that $0<\varepsilon_{n}<1$ and $\varepsilon_{n} \rightarrow 0$. It is clear that for each $n$ there must exist $y_{n} \in\left(x_{0}-\varepsilon_{n}, x_{0}\right)$ such that $\mu\left[0, y_{n}\right] \leqq \varepsilon_{n}$, which clearly implies that $\mu\left[0, x_{0}\right)=0$. It follows similarly that $\mu\left(a_{0}, 1\right]=0$ and we can conclude that $\mu$ is the "point mass" at $x_{0}$, thus proving that $L_{\left\langle x_{0}, \alpha_{0}\right)}$ is extreme.

To prove necessity of (ii), assume that one of the conaitions, say the first, is violated. This means that there is an $\varepsilon>0$ such that

$$
|\arg \alpha| \geqq \frac{\pi}{2}+\varepsilon \text { a.e }
$$

on $\left(x_{0}-\varepsilon, x_{0}\right)$. If we define $\mu\left(\left\{x_{0}-\varepsilon\right\}\right)=l, \mu\left(\left\{x_{0}\right\}\right)=1-l$ and $\mu=0$ elsewhere, where $0<l<\varepsilon$, we see that $|g| \leqq 1$ and $g \neq \alpha_{0}$, which means that $L_{\left\langle x_{0}, \alpha_{0}\right)}$ is not extreme. The necessity of the other condition is proved similarly.

Since the above proof works equally well when $x_{0}=0$ or 1 , Lemma 2.1 has been completely proved.

We do not really need the following result for later use, but since it is implicitly assumed in [1], we thought it worthwhile to include a proof.

LEMMA 2.2. If $\alpha, \beta$ satisfy the conditions of Lemma 2.1 at the points $x, y$ respectively, then $L_{(x, \alpha)}=L_{(y, \beta)}$ if and only if $x=y$ and $\alpha=\beta$ a.e.

Proof. We may assume that $x \leqq y$ and that $x$ and $y$ are interior points of $I$ as the following proof may be easily modified otherwise. By hypothesis, for all $f \in \mathscr{A} \mathscr{C}(I)$,

$$
f(x)+\int_{I} f^{\prime}(t) \bar{\alpha}(t) d t=f(y)+\int_{I} f^{\prime}(t) \bar{\beta}(t) d t
$$

or

$$
\int_{I} f^{\prime}(t)\left(\bar{\alpha}(t)-\bar{\beta}(t)-\chi_{[x, y]}(t)\right) d t=0
$$

and hence

$$
\bar{\alpha}(t)-\bar{\beta}(t)=\chi_{[x, y]}(t) \text { a.e. }
$$

$\left(\chi_{[x, y]}(t)\right.$ denotes the characteristic function of the interval $\left.[x, y]\right)$. This means that

$$
\alpha=\beta \text { a.e on } I-[x, y]
$$


and

$$
\alpha=1+\beta \text { a.e. on }[x, y] \text {. }
$$

It is easily seen that the last equation implies

$$
|\arg \alpha|=\frac{\pi}{3} \text { a.e. on }[x, y],
$$

which is impossible in view of condition (ii) of Lemma 2.1 unless $x=y$. This proves the lemma.

We can now turn to the question of the determination of all isometries of $\mathscr{A} \mathscr{C}(I)$. Let $\mathrm{T}$ be a (linear) isometry of $\mathscr{A} \mathscr{C}(I)$.

The following lemma occurs also in [1: page 221].

Lemma 2.3. $T(1)$ is the constant function $e^{i \theta}, \theta \in[-\pi, \pi]$.

Proof. The adjoint map $T^{*}$ carries the extreme functionals of

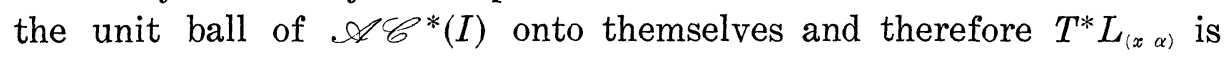
of the form $e^{i \eta} L_{(y, \beta)}, \eta \in[-\pi, \pi]$. Therefore,

$$
\left|(T 1)(x)+\int_{I}(T 1)^{\prime} \bar{\alpha} d \lambda\right|=1 .
$$

If we fix $x$ and vary $\alpha$ over all functions satisfying the conditions of Lemma 2.1, then the points

$$
(T 1)(x)+\int_{I}(T I)^{\prime} \bar{\alpha} d \lambda
$$

obviously describe the disc in the complex plane with centre $(T 1)(x)$ and radius $\int_{I}\left|(T 1)^{\prime}\right| d \lambda$, and this is obviously impossible unless $(T 1)^{\prime}=$ 0 a.e., which implies that $(T 1)(x)=e^{i \theta}$ for all $x \in I,(\theta \in[-\pi, \pi)]$.

We may therefore assume without loss of generality that $T(1)=$ 1. For the statement and proof of Lemma 2.4 below, we fix a point $x \in I$ and a function $\alpha_{x} \in L^{\infty}(I)$ defined by $\alpha_{x}(y)=1$ for $x-\varepsilon<y<x$ (if $x \neq 0$ ), $\alpha_{x}(y)=-1$ for $x<y<x+\varepsilon$ (if $x \neq 1$ ) and $\alpha_{x}(y)$ arbitrary at other points $y$ with the only restriction that $\left|\alpha_{x}(y)\right|=1$. By Lemma 2.1, the functionals $L_{\left(x, z \alpha_{x}\right)}$ are extreme for all $z \in C_{0}$,

$$
C_{0}=\left\{z \in \mathbf{C}:|z|=1,|\arg z| \leqq \frac{\pi}{2}\right\} .
$$

$T$ being an isometry, the adjoint map $T^{*}$ carries the extreme functionals $L_{\left(x, z \alpha_{x}\right)}$ into extreme functionals $L_{\left(y_{z}, \beta_{z}\right)}$ where the $\beta_{z}$ satisfy the conditions of Lemma 2.1 at the points $y_{z}$. We now prove the crucial 
Lemma 2.4. For a certain fixed $z_{1} \in C_{0}$,

$$
y_{z}=y_{z_{1}} \text { and } \beta_{z}=\frac{z}{z_{1}} \beta_{z_{1}}
$$

for all $z \in C_{0}$.

Proof. Assume that the set $\left\{\mathrm{y}_{z}: z \in C_{0}\right\}$ has a point of condensation $y_{z_{1}}$, say. (If this set has no points of condensation, then clearly it is countable, hence some $y_{z_{1}}$ is assumed infinitely often and as will be evident, the following proof is rendered easier.)

Since $T^{*} L_{\left(x, z \alpha_{x}\right)}=L_{\left(y_{z}, \beta_{z}\right)}$, we have

$$
\begin{aligned}
(*)(T f)(x)+\bar{z} \int_{I}(T f)^{\prime}(t) \bar{\alpha}_{x}(t) d t & =f\left(y_{z}\right)+\int_{I} f^{\prime}(t) \bar{\beta}_{z}(t) d t \\
& =f(0)+\int_{I}\left(\chi_{z}(t)+\bar{\beta}_{z}(t)\right) f^{\prime}(t) d t,
\end{aligned}
$$

for all $f \in \mathscr{A} \mathscr{C}(I), \chi_{z}$ denoting the characteristic function of the interval $\left[0, y_{z}\right]$.

We fix, for the moment, a point $z_{2} \in C_{0}$. For any $z \in C_{0}$, we can find $a_{1}, a_{2} \in \mathbf{C}$ such that

$$
a_{1}+a_{2}=1
$$

and

$$
a_{1} z_{1}+a_{2} z_{2}=z .
$$

Hence $(*)$ can be written as

$$
\begin{aligned}
& \bar{a}_{1}\left((T f)(x)+\bar{z}_{1} \int_{I}(T f)^{\prime} \bar{\alpha}_{x} d t\right)+\bar{a}_{2}\left((T f)(x)+\bar{z}_{2} \int_{I}(T f)^{\prime} \bar{a}_{x} d t\right) \\
& \quad=f(0)+\int_{I}\left(\chi_{z}+\bar{\beta}_{z}\right) f^{\prime} d t
\end{aligned}
$$

or,

$$
\begin{aligned}
& \bar{a}_{1}\left(f(0)+\int_{I}\left(\chi_{z_{1}}+\bar{\beta}_{z_{1}}\right) f^{\prime} d t\right)+\bar{a}_{2}\left(f(0)+\int\left(\chi_{z_{2}}+\bar{\beta}_{z_{2}}\right) f^{\prime} d t\right) \\
& \quad=f(0)+\int\left(\chi_{z}+\bar{\beta}_{z}\right) f^{\prime} d t .
\end{aligned}
$$

We can therefore assert that

$$
\chi_{z}+\bar{\beta}_{z}=\bar{a}_{1}\left(\chi_{z_{1}}+\bar{\beta}_{z 1}\right)+\bar{\alpha}_{2}\left(\chi_{z_{2}}+\bar{\beta}_{z_{2}}\right) \text { a.e. }
$$

or,

$$
\frac{\chi_{z}-\chi_{z_{1}}+\bar{\beta}_{z}}{\bar{\beta}_{z_{1}}}=\bar{a}_{1}+\bar{a}_{2} \frac{\chi_{z_{2}}+\bar{\beta}_{z_{2}}-\chi_{z_{1}}}{\bar{\beta}_{z_{1}}} \text { a.e. }
$$


Since (if $z \neq z_{1}$ and $\neq z_{2}$ ) $|\lambda|=1$ and $\left|a_{1}+a_{2} \lambda\right|=1$ imply that $\lambda=1$ or $z_{2} / z_{1}$, we immediately see that

$$
\frac{\chi_{z_{2}}+\bar{\beta}_{z_{2}}-\chi_{z_{1}}}{\bar{\beta}_{z_{1}}}=1 \text { or } \frac{\bar{z}_{2}}{\bar{z}_{1}} \text { a.e. on }\left\{s: \chi_{z}(s)=\chi_{z_{1}}(s)=\chi_{z_{2}}(s)\right\} \text {. }
$$

Choosing $z$ such that $y_{z}$ is arbitrarily close to $y_{z_{1}}$, we deduce that

$$
\frac{\chi_{z_{2}}-\chi_{z_{1}}+\bar{\beta}_{z_{2}}}{\bar{\beta}_{z_{1}}}=1 \text { or } \frac{\bar{z}_{2}}{\bar{z}_{1}} \text { on }\left\{s: \chi_{z_{1}}(s)=\chi_{z_{2}}(s)\right\} \text {. }
$$

Therefore,

$$
\frac{\chi_{z}-\chi_{z_{1}}+\bar{\beta}_{z}}{\bar{\beta}_{z_{1}}}=1 \text { or } \frac{\bar{z}}{\bar{z}_{1}} \text { on }\left\{s: \chi_{z_{1}}(s)=\chi_{z_{2}}(s)\right\} .
$$

The left-hand side being independent of $z_{2}$, we can now vary $z_{2}$ such that $y_{z_{2}}$ is arbitrarily close to $y_{z_{1}}$ to conclude that

$$
\frac{\chi_{z}-\chi_{z_{1}}+\bar{\beta}_{z}}{\bar{\beta}_{z_{1}}}=1 \text { or } \frac{\bar{z}}{\bar{z}_{1}} \text { a.e. on } I \text {. }
$$

Let $E$ be the set where

$$
\frac{\chi_{z}-\chi_{z_{1}}+\bar{\beta}_{z}}{\bar{\beta}_{z_{1}}}=1
$$

Then it is easy to see that the (measurable) set $E$ is independent of $z$. Reverting to the the original equation $(*)$, we can write

$$
\begin{aligned}
& (T f)(x)+\bar{z} \int_{I}(T f)^{\prime} \bar{\alpha}_{x} d t=f(0)+\int_{E}\left(\chi_{z_{1}}+\bar{\beta}_{z_{1}}\right) f^{\prime} d t \\
& \quad+\int_{I-E}\left(\chi_{z_{1}}+\frac{\bar{z}}{\bar{z}_{1}} \bar{\beta}_{z 1}\right) f^{\prime} d t
\end{aligned}
$$

for all $f \in \mathscr{C} \mathscr{C}(I)$ and for all $z \in C_{0}$. This being an identity in $z$,

$$
\int_{I}(T f)^{\prime} \bar{\alpha}_{x} d t=\frac{1}{z_{1}} \int_{I-E} f^{\prime} \bar{\beta}_{z_{1}} d t
$$

which immediately implies

$$
\int_{I}\left|(T f)^{\prime}\right| d t \leqq \int_{I-E}\left|f^{\prime}\right| d t \leqq \int_{I}\left|f^{\prime}\right| d t,
$$

(since $\alpha_{x}$ is virtually at our choice, the only restrictions on it being in a neighbourhood of $x$ ). On applying this result to the isometry $T^{-1}$, we have $\left\|f^{\prime}\right\|_{1} \leqq\left\|(T f)^{\prime}\right\|_{1}$ and hence

$$
\left\|f^{\prime}\right\|_{1}=\left\|(T f)^{\prime}\right\|_{1}=\int_{I-E}\left|f^{\prime}\right| d t \text {. }
$$


This says that $m(E)=0$, which means that

$$
\frac{\chi_{z}-\chi_{z_{1}}+\bar{\beta}_{z}}{\bar{\beta}_{z_{1}}}=\frac{\bar{z}}{\bar{z}_{1}} \text { a.e. }
$$

Therefore,

$$
\chi_{z}+\bar{\beta}_{z}=\chi_{z_{1}}+\frac{\bar{z}}{\bar{z}_{1}} \bar{\beta}_{z_{1}} \text { a.e. }
$$

and we can conclude (see proof of Lemma 2.2) that

$$
y_{z}=y_{z_{1}} \text { and } \beta_{z}=\frac{z}{z_{1}} \beta_{z_{1}} \text { a.e. }
$$

This proves the lemma.

We continue with the notations introduced just prior to the statement of Lemma 2.4. Let $\tau$ be the image under $T$ of the identity map of $I$ onto itself. Then

$$
T^{*} L_{\left(x, z \alpha_{x}\right)}=L_{\left(y_{z}, \frac{z}{z_{1}} \beta_{z_{1}}\right)} \text { (Lemma 2.4) }
$$

implies that

$$
\tau(x)+\bar{z} \int_{I} \tau^{\prime} \alpha_{x} d t=y_{z_{1}}+\frac{\bar{z}}{\bar{z}_{1}} \int_{I} \bar{\beta}_{z_{1}} d t
$$

and

$$
(T f)(x)+\bar{z} \int_{I}(T f)^{\prime} \bar{\alpha}_{x} d t=f\left(y_{z_{1}}\right)+\frac{\bar{z}}{\bar{z}_{1}} \int_{I} f^{\prime} \bar{\beta}_{z_{1}} d t
$$

for all $z \in C_{0}$ and all $f \in \mathscr{A} \mathscr{C}(I)$. Since these are identities in $z$, we deduce that

$$
\begin{gathered}
\tau(x)=y_{z_{1}} \\
\text { and }(T f)(x)=f(\tau(x))
\end{gathered}
$$

for all $f \in \mathscr{A} \mathscr{C}(I)$. These imply that $\tau$ is a continuous one-one map of $I$ into $I$, which means that $\tau$ is monotone and because $\int_{I}\left|\tau^{\prime}\right| d t=1$ (since $T$ preserves $L^{\prime}$ norms), we see easily that it is an onto map. We have therefore proved

Theorem 2.5. Any linear isometry $T$ of $A C(I)$ is of the form

$$
(T f)(x)=e^{i 0} f(\tau(x)),
$$

where $\tau$ is a monotone absolutely continuous mapping of I onto itself and $\theta$ is a constant in $[-\pi, \pi]$. Conversely, any transformation $T$ of the above form is a linear isometry of $\mathscr{A} \mathscr{C}(I)$. 
3. The algebra $\left.\mathscr{L}_{\%} / \mathrm{I}\right)$. For $f \in \mathscr{L}_{\%} /(I),\|f\|=\|f\|_{\infty}+\left\|f^{\prime}\right\|_{\infty}$. Throughout this section we let $\mathscr{C}$ denote the maximal ideal space of $L^{\infty}(I)$ with the $w^{*}$ topology. Let $X$ be the space $I \times \mathscr{C l} \times T$ with the product topology, where $T$ denotes the unit circle $\{z \in \mathbf{C}:|z|=1\}$ in the complex plane. Under the isometric map $f \rightarrow \widetilde{f}$,

$$
\widetilde{f}(x, m, z)=f(x)+z \hat{f}^{\prime}(m),
$$

$\mathscr{L} \%(I)$ is realised as a closed subspace of $\mathscr{C}(X) .{ }^{1}$ Clearly, therefore, all the extreme functionals in the unit ball of $\mathscr{L}_{\%}^{*} *(I)$ are contained in

$$
\left\{e^{i \eta} L_{(x, m, z)}:(x, m, z) \in X\right\} .
$$

where $L_{x, m, z}(f)=f(x)+z \hat{f}^{\prime}(m)$ for all $f \in \mathscr{L}_{y / 2}(I)$.

LEMMA 3.1. Every $L_{\left(x_{0}, m_{0}, z_{0}\right)}\left(\left(x_{0}, m_{0}, z_{0}\right) \in I \times \mathscr{C l} \times T\right)$ is extreme in the unit ball of $\mathscr{L}_{\text {/p }}^{*}(I)$.

Proof. $L_{x_{0}, m_{0}, z 0}$ being a linear functional defined on a subspace of $C(X)$ can be extended with preservation of norm to the whole of $C(X)$ by the Hahn-Banach theorem. Since $\left\|L_{\left(x_{0}, m_{0}, z_{0}\right)}\right\|=L_{\left\{x_{0}, m_{0}, z_{0}\right)}$ $(1)=1$, we see that $L_{\left(x_{0}, m_{0}, z_{0}\right)}$ is represented by a nonnegative measure $\mu$ on $X$ and hence

$$
f\left(x_{0}\right)+z_{0} \hat{f}^{\prime}\left(m_{0}\right)=\int_{X} \tilde{f} d \mu \cdots \cdots
$$

for all $f \in \mathscr{S}_{/ p}(I)$.

We recall that $\pi: \mathscr{C} \rightarrow I$ is the continuous projection defined by $\pi(m)=m(t)$, where $m$ is a multiplicative linear functional on $L^{\infty}(I)$ and $t$ is the identity mapping of $I$ onto $I$. (See [3; page 171].) It is easy to see that given any neighbourhood $N$ of $\pi\left(m_{0}\right)$ and any $\varepsilon>0$, we can find a $\mathscr{C}^{1}$ function $f_{1}$ such that

$$
\left\|f_{1}\right\|_{\infty}<\varepsilon,\left\|f_{1}^{\prime}\right\|_{\infty}=1, f_{1}^{\prime}\left(\pi\left(m_{0}\right)\right)=1
$$

and $f_{1}$ vanishes outside $N$.

It follows from (1) that

$$
1-\varepsilon \leqq\left|\int_{X} \tilde{f}_{1} d \mu\right| \leqq(1+\varepsilon) \mu\left((N \times \mathscr{C} \times T) \cup\left(I \times \pi^{-1}(N) \times T\right)\right) .
$$

As $N$ tends to $\pi\left(m_{0}\right)$ and $\varepsilon \rightarrow 0$, we get

$$
\mu\left(\left(\pi\left(m_{0}\right) \times \mathscr{C} \times T\right) \cup\left(I \times \pi^{-1}\left(\pi\left(m_{0}\right)\right) \times T\right)\right)=1,
$$

which means that the support of $\mu$ is concentrated on the set

$1 \hat{f}^{\prime}$ denotes the Gelfand transform of $f .^{\prime}$. 


$$
\left(\pi\left(m_{0}\right) \times \mathscr{C} \times T\right) \cup\left(I \times \pi^{-1}\left(\pi\left(m_{0}\right) \times T\right) .\right.
$$

Since we can choose the function $f_{1}$ defined above so that $f_{1}\left(\pi\left(m_{0}\right)\right)=0$, it follows by repeating the argument just given that

$$
\mu\left(I \times \pi^{-1}\left(\pi\left(m_{0}\right)\right) \times T\right)=1 \cdots \cdots(2) .
$$

We now claim now that a $\mathscr{C}^{1}$ function $f_{2}$ can be found such that

$$
f_{2}\left(x_{0}\right)=1,\left\|f_{2}\right\|_{\infty}=1, f_{2}^{\prime}\left(\pi\left(m_{0}\right)\right)=0 \text { and }
$$

$\left|f_{2}(x)\right|<f_{2}\left(x_{0}\right)=1$ for all $x \neq x_{0}$. This is seen as follows.

(i) If $\pi\left(m_{0}\right)=x_{0}$, we simply let $f_{2}$ be a $\mathscr{C}^{1}$ function which peaks exactly at $x_{0}$; consequently; $f_{2}^{\prime}\left(x_{0}\right)=0$.

(ii) If $\pi\left(m_{0}\right) \neq x_{0}$, we let $f_{2}$ be a peaking $\mathscr{C}^{1}$ function at $x_{0}$ which vanishes in a small neighbourhood of $y_{0}$ that does not contain $\pi\left(m_{0}\right)$.

Hence, on using (2), we deduce that $\mu$ must be concentrated on the "peak set" of $f_{2}$, viz. $x_{0} \times \pi^{-1}\left(\pi\left(m_{0}\right)\right) \times T$. Therefore, for all $f^{\prime} \in L^{\infty}(I)$.

$$
z_{0} \hat{f}^{\prime}\left(m_{0}\right)=\int_{P} z \hat{f}^{\prime} d \mu
$$

where $P$ denotes the set $\left(x_{0} \times \pi^{-1}\left(\pi\left(m_{0}\right)\right) \times T\right)$. Setting $f^{\prime} \equiv 1$, we see that support of $\mu$ is contained in $x_{0} \times \pi^{-1}\left(\pi\left(m_{0}\right)\right) \times z_{0}$ whence it follows that

$$
\hat{f}^{\prime}\left(m_{0}\right)=\int_{P} \hat{f}^{\prime} d \mu .
$$

This being true for all $f^{\prime} \in L^{\infty}(I)(\equiv \mathscr{C}(\mathscr{C l}))$, we finally conclude that $\mu$ is the "point mass" at $\left(x_{0} m_{0} z_{0}\right)$. This completes the proof that

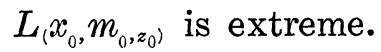

Following the pattern of argument of $\S 2$, we now prove

Lemma 3.2. $T(1)$ is the constant function $e^{i \theta}, \theta \in[-\pi, \pi]$.

Proof. Since $T^{*}$ is also an isometry,

$$
T^{*} L_{(x, m, z)}=e^{i \eta} L_{\left(x_{1}, m_{1}, z_{1}\right)}
$$

where $\eta \in[-\pi, \pi]$. We therefore have

$$
\left|(T 1)(x)+z(\widehat{T 1})^{\prime}(m)\right|=1
$$

for all $(x, m, z) \in X$. This is clearly impossible unless $(T 1)^{\prime}=0$ a.e. which implies that $T 1$ is a constant function of modulus one.

We may thus assume without loss of generality that $T 1=1$. Then, since $T^{*} L_{(x, m, z)}=L_{\left(x_{1}, m_{1}, z_{1}\right)}$,

$$
(x, m, z) \rightarrow\left(x_{1}, m_{1}, z_{1}\right)
$$


is a homeomorphism of $X$ onto itself. If $\tau$ denotes the image under $T$ of the identity map $t$ of $I$ onto itself then,

$$
\tau(x)+z \hat{\tau}^{\prime}(m)=x_{1}+z_{1} .
$$

Now the points $x_{1}+z_{1}$ as $\left(x_{1}, z_{1}\right)$ vary over all of $I \times T$, describe the region in the complex plane bounded by circles at centres 0 and 1 , each of unit radius, and their common tangents. It is clear that $\tau(x)+z \hat{\tau}^{\prime}(m)$ can belong to this region only if

$$
\left\|\tau^{\prime}\right\|_{\infty} \leqq 1
$$

(We recall that $\left\|\hat{\tau}^{\prime}\right\|_{\infty}=\left\|\tau^{\prime}\right\|_{\infty}$. )

Also, there must exist points $\left(x^{\prime}, m^{\prime}, z^{\prime}\right),\left(x^{\prime \prime}, m^{\prime \prime}, z^{\prime \prime}\right)$ in $X$ such that

Therefore,

$$
\tau\left(x^{\prime}\right)+z^{\prime} \hat{\tau}^{\prime}\left(m^{\prime}\right)=-1, \tau\left(x^{\prime \prime}\right)+z^{\prime \prime} \hat{\tau}^{\prime}\left(m^{\prime \prime}\right)=2 .
$$

$$
\left|\tau\left(x^{\prime \prime}\right)-T\left(x^{\prime}\right)\right| \geqq 3-\left|\tau^{\prime}\left(m^{\prime \prime}\right)-\tau^{\prime}\left(m^{\prime}\right)\right| \geqq 1
$$

and this is possible only when $x^{\prime}, x^{\prime \prime}$ are end points of $I$ and therefore $\left|\tau^{\prime}\right| \equiv 1$ a.e. A moment's reflection will show that $\tau$ (and hence $\tau^{\prime}$ ) $\tau \equiv t$ or $1-t$. Hence $(*)$ says that either,

is real and hence we must have either $\tau^{\prime} \equiv 1$ or -1 . Consequently,

$$
x_{1}+z_{1} \equiv x+z
$$

or,

$$
x_{1}+z_{1} \equiv 1-x-z .
$$

We now claim that these relations imply $x_{1} \equiv x, z_{1} \equiv z$ and $x_{1} \equiv$ $1-x, z_{1} \equiv-z$ respectively. For, let $x_{1}+z_{1} \equiv x+z$. Then $\operatorname{Im} z_{1}=$ $\operatorname{Im} z$ and therefore, $\operatorname{Re} z_{1}=\operatorname{Re} z$ or-Re $z$. But when $\operatorname{Im} z=0$, i.e. when $\operatorname{Re} z= \pm 1$ we have $\operatorname{Re} z_{1}=\operatorname{Re} z$. Hence on the set $\operatorname{Re} z \neq 0$, we have $z_{1} \equiv z$ and $x_{1} \equiv x$ and therefore $x_{1} \equiv x, z_{1} \equiv z$ everywhere. The second case is treated similarly and this proves our claim.

Now we shall prove that for all $f \in \mathscr{L}_{\mathrm{g} / 2}(I), T f(x)=f(x)$ or $f(1-x)$ according as $x_{1} \equiv x$ or $x_{1} \equiv 1-x$. Let us, for instance, take the second case when $x_{1} \equiv 1-x$ and $z_{1} \equiv z$, the first case being treated similarly. We have

$$
(T f)(x)+z \widehat{(T f})^{\prime}(m)=f(1-x)-z f^{\prime}\left(m_{1}\right)
$$

for all $f \in \mathscr{L}_{\text {g/ }}(I)$. Since $\mathscr{C}$ is totally disconnected, (see [3; page 170]) we see easily that if we fix $m$ in the above equation, then $m_{1}$ is also fixed in the sense that $m_{1}$ is independent of $x$ and $z$. Hence fixing $x$ and $m$ and varying $z$ on $T$, we see that the right hand side of the above equation represents a circle with centre at $f(1-x)$ and the the left hand side a circle with centre at $(T f)(x)$, and since 
these circles are identical as sets, we can conclude that

$$
(T f)(x)=f(1-x) \text {. }
$$

On summarising the above discussion, we get the following

THEOREM 3.3. All isometries $T$ of $\mathscr{L}_{\text {gin }}(I)$ are of the form

$$
(T f)(x)=e^{i \theta} f(x)
$$

or

$$
(T f)(x)=e^{i \theta} f(1-x)
$$

and conversely, $\theta \in[-\pi, \pi]$.

REMARKS 1. If we let

$$
\mathscr{A} \mathscr{C}^{p}(I)=\left\{f \in \mathscr{C}(I): f^{\prime} \text { exists a.e., } f^{\prime} \in L^{p}(I)\right\},
$$

then $p=1$ (resp. $\infty$ ) gives the space $\mathscr{C} \mathscr{C}(I)$ (resp. $\mathscr{L}$ g/ $(I))$. So far as we know, the isometries of $\mathscr{A} \mathscr{C}^{p}(I), 1<p<\infty$, have never been determined. One of the main difficulties seems to be in finding a characterization, analogous to Lemma 2.1, of the extreme functionals in the unit ball of the dual of $\mathscr{C} \mathscr{C}^{p}(I)$.

2. If $X$ is any compact metric space with metric $d$, let

$$
\mathscr{L}_{\mathscr{q} / 2}\left(X, d^{\alpha}\right)=\left\{f \in \mathscr{C}(X):\|f\|_{d^{\alpha}}=\sup _{\substack{x, y \in X \\(x \neq y)}} \frac{|f(x)-f(y)|}{d^{\alpha}(x, y)}<\infty\right\}
$$

and

$$
\text { liph }\left(X, d^{\alpha}\right)=\left\{f \in \mathscr{L}_{y / 2}\left(X, d^{\alpha}\right): \lim _{d(x, y) \rightarrow 0} \frac{|f(x)-f(y)|}{d^{\alpha}(x, y)}=0\right\}
$$

both provided with the norm

$$
\|f\|=\|f\|_{\infty}+\|f\|_{\alpha^{\alpha}} \text {. }
$$

These spaces are Banach algebras under the norms just defined. (See [6]). It would be interesting to know whether the results of $\S 3$ are valid for these algebras, viz. whether all their isometries are induced by the isometries of the metric space $X$. An affirmative answer would not be surprising because this indeed is the case for the norm

$$
\|f\|=\max \left(\|f\|_{\infty},\|f\|_{\alpha^{\alpha}}\right) \text {. }
$$

(See [4] and [5]).

4. The algebra $\mathscr{C}^{1}(\mathrm{I})$. The norm is the same as that for $(I)$,

$$
\|f\|=\|f\|_{\infty}+\left\|f^{\prime}\right\|_{\infty},\left(f \in \mathscr{C}^{1}(J)\right) .
$$


Following what is by now a standard argument, we embed $\mathscr{C}^{1}(I)$ as a subspace of $\mathscr{C}(I \times I \times T)$ in the obvious way and we can prove by the methods of the last section that all the "point evaluations",

$$
L_{(x, y, z)}
$$

are extreme in the unit ball of $\left(\mathscr{C}^{1}(I)\right)^{*}$, that for an isometry $T$ of $\mathscr{C}^{1}(I), T(1)$ is a constant function of modulus one and futher that if

$$
\left.T^{*} L_{(x, y, z)}=L_{\left(x_{1}, y_{1}, z_{1}\right)} \text { (assuming } T(1)=1\right),
$$

then, either

$$
x_{1} \equiv x \text { and } z_{1} \equiv z
$$

or

$$
x_{1} \equiv 1-x \text { and } z_{1} \equiv-z .
$$

Assuming the second possibility, we therefore have

$$
(T f)(x)+z(T f)^{\prime}(y)=f\left(x_{1}\right)-z f^{\prime}\left(y_{1}\right), \forall f \in \mathscr{C}^{1}(I) .
$$

At this point, we have to proceed in a manner different from that of $\S 3$ since the disconnectedness of $\mathscr{C l}$ is not available for us to conclude that as a function, $y_{1}$ is independent of $x$ and $z$. But in fact we can prove that in this instance, $y_{1}=1-y$. Considering the function $f(x)=x^{2},(x \in I)$ and its image $g$ under $T$, we have from the above equation,

$$
g(x)+z g^{\prime}(y)=(1-x)^{2}-2 z y_{1}
$$

and therefore

$$
2 y_{1}=\frac{(1-x)^{2}-g(x)}{z}-g^{\prime}(y) .
$$

$y_{1}$ being real-valued, this says that for fixed $(x, y), y_{1}$ is a meromorphic function with real boundary values!

Hence $g(x)=(1-x)^{2}$ and we see that

$$
y_{1} \equiv 1-y \text {. }
$$

We can prove similarly that $y_{1} \equiv y$ when $x_{1} \equiv x$ and $z_{1} \equiv z$.

Now we can proceed exactly as we did in the last section to derive the following

THEOREM 4.1. Every isometry $T$ of $\mathscr{C}^{1}(I)$ is of the form

$$
(T f)(x)=e^{i \theta} f(x)
$$

or

$$
(T f)(x)=e^{i 0} f(1-x)
$$

and conversely, $\theta \in[-\pi, \pi]$. 
5. Isometries under more general norms. We propose to show now that the results of the preceding sections are valid for more general norms on the spaces concerned. Our discussion will be brief and we shall omit proofs since they are the same as before with minor modifications. We restrict attention to $\mathscr{L}_{\text {if }}(I)$, the treatment of the other spaces being similar.

It is well-known that in a topological vector space, there is a one-one correspondence between semi-norms and convex, symmetric neighbourhoods of the origin. Consequently, if $K$ is a given convex, symmetric neighbourhood of 0 in $\mathrm{C}^{2}$, there is a unique norm $N$ associated with it such that

$$
K=\left\{\left(z_{1}, z_{2}\right) \in \mathbf{C}^{2}: N\left(z_{1}, z_{2}\right) \leqq 1\right\} .
$$

We assume that $K$ has the following additional properties:

(i) if $\left(a_{1}, a_{2}\right) \in K$ then $\left(e^{i \theta} a_{1}, e^{i \varphi} a_{2}\right) \in K$ for all $\theta, \varphi \in[-\pi, \pi]$ and

(ii) the intersection of $K$ with the first quadrant of $R^{2}$ is a convex polytope, i.e. this intersection is a convex body with a finite number of extreme points.

It is an easy exercise to prove that if $K$ has the above properties then the compact set $K^{*}$ associated with the conjugate norm $N^{*}$ on $\mathrm{C}^{2}$,

$$
K^{*}=\left\{\left(z_{1}, z_{2}\right): N^{*}\left(z_{1}, z_{2}\right) \leqq 1\right\}
$$

inherits the same properties. We recall that

$$
N^{*}\left(z_{1}, z_{2}\right)=\max _{N\left(w_{1}, w_{2}\right) \leqq 1}\left|w_{1} z_{1}+w_{2} z_{2}\right| \cdot
$$

We will show that $\mathscr{L}_{\text {fp }}(I)$ equipped with the norm

$$
\|f\|_{N}=\max _{(x, m) \in I \times \mathscr{M}} N\left(f(x), \hat{f}^{\prime}(m)\right)
$$

has only the isometries coming from the functions $x$ and $1-x$ on $\mathrm{I}$. We note that the norm

$$
\|f\|=\|f\|_{\infty}+\left\|f^{\prime}\right\|_{\infty}
$$

of $\S 3$ is a particular case of the above, the sets $K$ and $K^{*}$ being $\left\{\left(z_{1}, z_{2}\right) \in \mathbf{C}^{2}:\left|z_{1}\right|+\left|z_{2}\right| \leqq 1\right\}$ and $\left\{\left(z_{1}, z_{2}\right): \max \left(\left|z_{1}\right|,\left|z_{2}\right|\right) \leqq 1\right\}$ respectively, and both these sets have the properties (i) and (ii) listed above. Now,

$$
\begin{aligned}
\|f\|_{N} & =\max _{(x, m) \in I \times \mathbb{N}} N\left(f(x), \hat{f}^{\prime}(m)\right) & \\
& =\max _{\left(x, m, a_{1}, a_{2}\right) \in I \times \mathscr{N} \times K^{*}}\left|a_{1} f(x)+a_{2} f^{\prime}(m)\right| & \\
& =\max _{\substack{1 \leq i \leq n \\
(x, m, z) \in I \times \mathbb{M} \times T}}\left|s_{i} f(x)+t_{i} z \hat{f}^{\prime}(m)\right| & \text { (by properties (i) and }
\end{aligned}
$$


where $g_{i} \geqq 0, t_{i} \geqq 0 \quad(i=1, \cdots n)$. Hence if we take $n$ copies of $I \times \mathscr{C} \times T$, the $i^{t h}$ copy being denoted by $(I \times \mathscr{C} \times T)_{i}$, and define $f$ on $(I \times \mathscr{C l} \times T)_{i}$ by

$$
\widetilde{f}(x, m, z)=s_{i} f(x)+t_{i} z \hat{f}^{\prime}(m),
$$

then the mapping $f \rightarrow \hat{f}$ clearly establishes an isometric isomorphism between $\mathscr{G} /(I)$ with norm $\|\cdot \cdot\|_{N}$ and a subspace of $\mathscr{C}(X)$,

$$
X=\bigcup_{i=1}^{n}(I \times \mathscr{L} \times T)_{i} .
$$

As before, we can prove,

LEMma 5.1. The functionals $\left\{e^{i \eta} L_{(x, m, z)}^{k}: \eta \in[-\pi, \pi],(x, m, z) \in X\right\}$ are all extreme in the unit ball of $\mathscr{L}_{/ 2}^{*}(I)$ and conversely, where for $f \in \mathscr{L}_{/ / 2}(I)$,

$$
L_{(x, m, z)}^{k}(f)=s_{k} f(x)+t_{k} f^{\prime}(m),(k=1, \cdots, n) .
$$

Because of property (ii) of $K^{*}$, it cannot happen that the extreme points of $K^{*} \cap R^{2}$ all lie on the $x$-axis or that they all lie on the $y$-axis. However, it may happen that some of them lie on the $x$-axis while the remaining ones are on the $y$-axis. It will be seen that in this case there are precisely four extreme points, symmetrically situated with respect to the origin, and that the norm $\|\cdots\|_{N}$ comes from the set

$$
K=\left\{\left(z_{1}, z_{2}\right) \in \mathbf{C}^{2}: \max \left(\left|z_{1}\right|,\left|z_{2}\right|\right) \leqq 1\right\} \text {. }
$$

We already know (from [4] and [5]) that the isometries of $\mathscr{L}_{\text {if }}(I)$ with this norm are induced by the functions $x$ and $1-x$. We may therefore assume that there is at least one pair $\left(s_{i}, t_{i}\right)$ with $s_{i}>0$, $t_{i}>0$. With this observation, one proves as before that $T(1)$ is a constant function, that $T(t)$ is $t$ or $1-t$ and finally one derives the following.

THEOREM 5.2. All the isometries of $\mathscr{L}_{\text {g/ }}(I)$ with the norm $\|\cdots\|_{N}$ are of the form

$$
(T f)(x)=e^{i \theta} f(x)
$$

or

$$
(T f)(x)=e^{i \theta} f(1-x)
$$

and conversely, $\theta \in[-\pi, \pi]$.

REMARKs. 1. Although norms of the type described above form a fairly large class, there are many others of an essentially different nature for which Theorem 5.1 may or may not be true. For example, 
we may ask whether Theorem 5.1 is valid for the norm

$$
\|f\|=\sqrt{\|f\|_{\infty}^{2}+\left\|f^{\prime}\right\|_{\infty}^{2}},\left(f \in \mathscr{L}_{/ 2}(I)\right),
$$

which comes from the set

$$
K=\left\{\left(z_{1}, z_{2}\right) \in \mathbf{C}^{2}:\left|z_{1}\right|^{2}+\left|z_{2}\right|^{2} \leqq 1\right\} \text {. }
$$

(Note that $K$ does not have property (ii)).

2. It is not true, however, that Theorem 5.1 is true for all norms equivalent to the norm used in §3. If we define, for $x_{0} \in I$,

$$
\|f\|_{1}=\max \left(\left|f\left(x_{0}\right)\right|,\left\|f^{\prime}\right\|_{\infty}\right),\left(f \in \mathscr{L}_{/ 2}(I)\right),
$$

then $\|\cdots\|_{1}$ is such a norm and it is easy to see that if $x_{0} \neq 1 / 2$ then the transformation $T$ defined on $\mathscr{L}_{y / 2}(I)$ by $(T f)(x)=f(1-x)$ is not an isometry. It is not hard to prove that for $\|\cdot\|_{1}$, all the isometries of $\mathscr{L}_{/ /}(I)$ are induced by homeomorphisms of the maximal ideal space $\mathscr{l l}$ of $L^{\infty}(I)$.

\section{REFERENCES}

1. M. Cambern, Isometries of certain Banach algebras, Studia Mathematica, 25 (1965), 217-225.

2. N. Dunford and J. Schwartz, Linear Operators (Part I), Inter since, New York, 1958.

3. K. Hoffman, Banach spaces of analytic Functions, Prentice-Hall, Inc. N.J., 1962.

4. T. M. Jenkins, Banach spaces of Lipschitz functions on an abstract metric space, Ph.D. Thesis, Yale University, 1967.

5. A. K. Roy, Extreme points and Linear isometries of the Banach space of Lipschitz functions, Canadian J. Math. 20 (1968). 1150-1164.

6. D. R. Sherbert, Banach algebras of Lipschitz functions, Pacific J. Math., 13 (1963) 1387-1399.

Received January 7, 1971.

Tata Institute of Fundamental Research

BOMbAY 5 


\section{PACIFIC JOURNAL OF MATHEMATICS}

\section{EDITORS}

H. SAMELSON

Stanford University

Stanford, California 94305

C. R. Hовву

University of Washington

Seattle, Washington 98105
J. DUGUNDJI

Department of Mathematics

University of Southern California

Los Angeles, California 90007

RICHARD ARENS

University of California

Los Angeles, California 90024

\section{ASSOCIATE EDITORS}
E. F. BECKENBACH
B. H. NeumanN
F. WOLF
K. YoSHIDA

\section{SUPPORTING INSTITUTIONS}

UNIVERSITY OF BRITISH COLUMBIA

CALIFORNIA INSTITUTE OF TECHNOLOGY

UNIVERSITY OF CALIFORNIA

MONTANA STATE UNIVERSITY

UNIVERSITY OF NEVADA

NEW MEXICO STATE UNIVERSITY

OREGON STATE UNIVERSITY

UNIVERSITY OF OREGON

OSAKA UNIVERSITY

UNIVERSITY OF SOUTHERN CALIFORNIA
STANFORD UNIVERSITY

UNIVERSITY OF TOKYO

UNIVERSITY OF UTAH

WASHINGTON STATE UNIVERSITY

UNIVERSITY OF WASHINGTON

AMERICAN MATHEMATICAL SOCIETY CHEVRON RESEARCH CORPORATION NAVAL WEAPONS CENTER 


\section{Pacific Journal of Mathematics}

\section{Vol. 38, No. $1 \quad$ March, 1971}

Bruce Alan Barnes, Banach algebras which are ideals in a Banach algebra ..... 1

David W. Boyd, Inequalities for positive integral operators............... 9

Lawrence Gerald Brown, Note on the open mapping theorem .............. 25

Stephen Daniel Comer, Representations by algebras of sections over Boolean

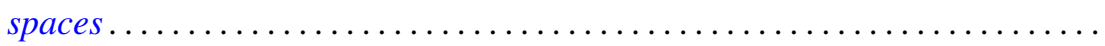

John R. Edwards and Stanley G. Wayment, On the nonequivalence of

conservative Hausdorff methods and Hausdorff moment sequences ........

P. D. T. A. Elliott, On the limiting distribution of additive functions $(\bmod 1) \ldots \ldots$

Mary Rodriguez Embry, Classifying special operators by means of subsets

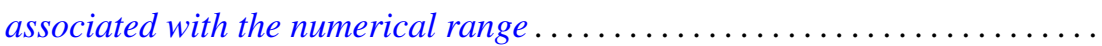

Darald Joe Hartfiel, Counterexamples to a conjecture of G. N. de Oliveira ......

C. Ward Henson, A family of countable homogeneous graphs...............

Satoru Igari and Shigehiko Kuratsubo, A sufficient condition for

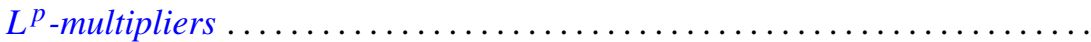

William A. Kirk, Fixed point theorems for nonlinear nonexpansive and

generalized contraction mappings............................

Erwin Kleinfeld, A generalization of commutative and associative rings ...... 95

D. B. Lahiri, Some restricted partition functions. Congruences modulo $11 \ldots \ldots 103$

T. Y. Lin, Homological algebra of stable homotopy ring $\pi *$ of spheres ....... 117

Morris Marden, A representation for the logarithmic derivative of a meromorphic function...........................

John Charles Nichols and James C. Smith, Examples concerning sum properties for metric-dependent dimension functions . .

Asit Baran Raha, On completely Hausdorff-completion of a completely

Hausdorff space.

M. Rajagopalan and Bertram Manuel Schreiber, Ergodic automorphisms and affine transformations of locally compact groups..........

N. V. Rao and Ashoke Kumar Roy, Linear isometries of some function

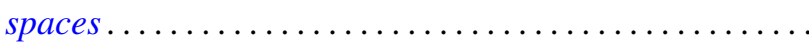

William Francis Reynolds, Blocks and F-class algebras of finite groups

Richard Rochberg, Which linear maps of the disk algebra are multiplicative ...

Gary Sampson, Sharp estimates of convolution transforms in terms of decreasing

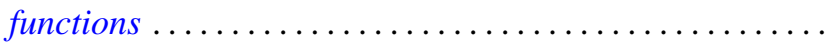

Stephen Scheinberg, Fatou's lemma in normed linear spaces

Ken Shaw, Whittaker constants for entire functions of several complex

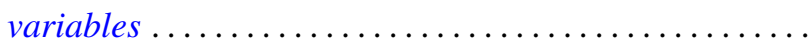

James DeWitt Stein, Two uniform boundedness theorems................ 251

$\mathrm{Li} \mathrm{Pi} \mathrm{Su,} \mathrm{Homomorphisms} \mathrm{of} \mathrm{near-rings} \mathrm{of} \mathrm{continuous} \mathrm{functions} \mathrm{.} \mathrm{.............} 261$

Stephen Willard, Functionally compact spaces, $C$-compact spaces and mappings of minimal Hausdorff spaces....................... 